Aus dem Anatomischen Institut der Med. Fakultät, Univ. Okayama

(Vorstand: Prof. M. SEKI).

\title{
Veränderungen des Redoxpotentials des Harns während der Schwangerschaft.
}

\author{
娃娠時の酸還電位の変化. \\ Takashi SATOMI 里見堯.
}

[Eingegangen am 28. Juli 1953.]

Seit langem ist der Harn ein günstiger Gegenstand der Untersuchungen des Stoffwechsels in der Schwangerschaft. Es handelt sich meist um eine qualitative und quantitative Bestimmung der ebemisehen Substanzen im Harn. Die frühzeitige Diagnose der Schwangerschaft ist auch auf biologischem Wege vorgenommen. Bezüglich der physikosbemischen Untersuchung des Harns der Schwangeren gibt es z. B. die von SCHIMIZU (1949) über die Kolloidreaktion. Im folgenden werden, um zur Histochemie indirekt beizutragen, die Veränderungen des Redoxpotentials des Schwangerschaftsharns, die der Verfasser mit Hilfe von rH-Indikatoren bestimmt hat, besprochen.

\section{Material und Methode.}

Das Redoxpotential des Harns von gesunden Schwangeren, d. h. frei von Fieberkrankheiten. Diabetes melitus, BASEDWscher Krankheit, wurde nach KUSUHARA (1950) bestimmt. Fs wurde nur Harn, der nicht lange Zeit in der Harnblase verweilt hatte, verwandt. "Bei der Entnahme des Harns hütete man sich vor einer Mischung des Ausflußes. Getrübter Harn wurde aus den Untersuchungen ausgeschlossen.

Das genaue Verfahren der rH-Bestimmung war wie folgt. Man füllte in eine Reihe Reagenzgläser je 2 co Harn, versetzte sie mit ein oder zwei Tropfen von verschiedenen $0.025 \%$ igen Indikatorlösungen und sah nach 15 Minuten nach einem halbentfärbten Indikator. Der $\mathrm{rH}-$ Wert dieses Indikators ist genau der des Harns. Die angewandten Indikatoren waren folgende 7 Farbstoffe. Methylenblau war von MERCK, und die übrigen waren alle von GRÜBLER. Die im folgenden geschriebene Zahl ist der rH-Wert des betreffenden Farbstoffes bei pH 7.0.

Malachitgrün 23, Methylgrün 21, Kristallviolett 20, Äthylviolett 19, Lichtgrün F. S. 18, Methylengrün 17, Methylenblau 14.

Es wurde zugleich das spezifische Gewicht des Harns mit dem Urometer und die Wasserstoffionenkonzentration mit den pH-Indikatoren bestimmt. 


\section{Ergebnisse der Untersuchungen.}

Das rH, ein Maß der Reduktionsintensität, des Harns wurde insgesamt an 150 Schwangeren- und 7 Nichtschwangeren bestimmt. Iie Resultate sind in Tabell 1 zusammengestellt. Aus der Tabelle sieht man, daß im Frühstadium der Schwangerschaft das $\mathrm{rH}$ mit wenigen Ausnahmen einen fast normalen Wert von 19-20 einhält. Mit dem Fortschreiten des Schwangerschaftsmonats erniedrigt sich der rH-Wert allmählich, und zwar im mittleren Stadium der Schwangerschaft beträgt derselbe hïufig 17-18, um schließlich im Endstadium in überwiegender Mehrzahl 17-18 zu zeigen.

Tabelle 1. rH des Schwangerenharns.

\begin{tabular}{|c|c|c|c|}
\hline & $\mathrm{rH}$ & $\mathrm{pH}$ & sp. Gew. \\
\hline Nichtschwangere & $\begin{array}{l}19 \\
20 \\
20 \\
19 \\
20 \\
20 \\
19\end{array}$ & $\begin{array}{l}7.0 \\
6.2 \\
6.4 \\
6.0 \\
6.0 \\
6.2 \\
6.2\end{array}$ & $\begin{array}{l}1.023 \\
1.020 \\
1.019 \\
1.022 \\
1.021 \\
1.017\end{array}$ \\
\hline 2. Mon. & $\begin{array}{l}20 \\
19 \\
20 \\
20 \\
20 \\
20 \\
18 \\
19 \\
19 \\
19\end{array}$ & $\begin{array}{l}5.6 \\
6.8 \\
6.2 \\
5.8 \\
5.2 \\
6.8 \\
7.0 \\
6.6 \\
6.6 \\
6.2\end{array}$ & $\begin{array}{l}1.024 \\
1.021 \\
1.018 \\
1.014 \\
1.016\end{array}$ \\
\hline 3. Mon. & $\begin{array}{l}19 \\
18 \\
19 \\
19 \\
20 \\
20 \\
19 \\
16 \\
20 \\
19 \\
19 \\
20 \\
19 \\
20 \\
19 \\
19 \\
19 \\
20 \\
19 \\
20 \\
19\end{array}$ & $\begin{array}{l}6.2 \\
7.0 \\
5.4 \\
6.6 \\
6.8 \\
5.8 \\
6.8 \\
6.4 \\
6.8 \\
6.8 \\
6.0 \\
6.0 \\
7.0 \\
6.6 \\
6.2 \\
6.4 \\
6.6 \\
6.2 \\
6.2 \\
6.4 \\
7.0\end{array}$ & $\begin{array}{l}1.010 \\
1.026 \\
1.012 \\
1.018 \\
1.017 \\
1.010 \\
1.015 \\
1.012 \\
1.023 \\
1.012\end{array}$ \\
\hline
\end{tabular}




\begin{tabular}{|c|c|c|c|}
\hline 4. Mon. & $\begin{array}{l}20 \\
16 \\
19 \\
18 \\
20 \\
19 \\
19 \\
20 \\
20 \\
17 \\
18 \\
16 \\
18 \\
19 \\
16 \\
18\end{array}$ & $\begin{array}{l}6.8 \\
6.4 \\
7.0 \\
6.6 \\
6.2 \\
7.0 \\
6.8 \\
6.2 \\
6.4 \\
6.8 \\
7.0 \\
5.4 \\
6.4 \\
6.6 \\
7.0 \\
6.4\end{array}$ & $\begin{array}{l}1.014 \\
1.010 \\
1.018 \\
1.010 \\
1.015 \\
1.010 \\
1.010\end{array}$ \\
\hline 5. Mon. & $\begin{array}{l}16 \\
20 \\
17 \\
17 \\
19 \\
19 \\
19 \\
17 \\
18 \\
18\end{array}$ & $\begin{array}{l}6.4 \\
7.0 \\
6.8 \\
6.8 \\
5.6 \\
5.2 \\
6.8 \\
6.0 \\
6.6 \\
7.0\end{array}$ & $\begin{array}{l}1.014 \\
1.016 \\
1.017 \\
1.028\end{array}$ \\
\hline 6. Mon. & $\begin{array}{l}16 \\
18 \\
18 \\
17 \\
18 \\
20 \\
17 \\
17 \\
18 \\
18 \\
19 \\
18\end{array}$ & $\begin{array}{l}7.0 \\
6.4 \\
6.2 \\
6.0 \\
6.6 \\
6.4 \\
6.6 \\
6.8 \\
6.8 \\
5.2 \\
6.2 \\
7.2\end{array}$ & $\begin{array}{l}1.022 \\
1.020 \\
1.018 \\
1.020 \\
1.025 \\
1.020 \\
1.012 \\
1.010\end{array}$ \\
\hline 7. Mon. & $\begin{array}{l}17 \\
17 \\
18 \\
16 \\
17 \\
18 \\
18 \\
19 \\
17 \\
17 \\
18 \\
18 \\
17\end{array}$ & $\begin{array}{l}7.0 \\
6.8 \\
7.2 \\
5.8 \\
6.8 \\
6.8 \\
7.0 \\
7.2 \\
6.6 \\
6.6 \\
6.2 \\
7.0 \\
7.0\end{array}$ & $\begin{array}{l}1.010 \\
1.020 \\
1.015 \\
1.016\end{array}$ \\
\hline 8. Mon. & $\begin{array}{l}17 \\
18 \\
17 \\
16 \\
16 \\
18\end{array}$ & $\begin{array}{l}6.4 \\
6.8 \\
6.8 \\
5.4 \\
7.0 \\
6.2\end{array}$ & $\begin{array}{l}1.010 \\
1.008\end{array}$ \\
\hline
\end{tabular}




\begin{tabular}{|c|c|c|c|}
\hline 8. Mon. & $\begin{array}{l}18 \\
17 \\
17 \\
17 \\
18 \\
17 \\
17 \\
17 \\
18 \\
17\end{array}$ & $\begin{array}{l}6.8 \\
6.8 \\
6.6 \\
7.0 \\
6.6 \\
6.8 \\
6.4 \\
7.0 \\
6.2 \\
6.4\end{array}$ & $\begin{array}{l}1.024 \\
1.012 \\
1.024\end{array}$ \\
\hline 9. Mon. & $\begin{array}{l}18 \\
17 \\
18 \\
17 \\
17 \\
16 \\
16 \\
17 \\
18 \\
17 \\
17 \\
19 \\
19 \\
16 \\
18 \\
17 \\
20\end{array}$ & $\begin{array}{l}6.4 \\
6.8 \\
6.6 \\
5.8 \\
5.6 \\
6.8 \\
6.4 \\
6.6 \\
5.8 \\
6.8 \\
6.6 \\
6.8 \\
6.6 \\
7.0 \\
6.6 \\
6.2 \\
6.2\end{array}$ & $\begin{array}{l}1.014 \\
1.010 \\
1.015 \\
1.002\end{array}$ \\
\hline 10. Mon. & $\begin{array}{l}17 \\
17 \\
17 \\
17 \\
16 \\
18 \\
16 \\
16 \\
17 \\
16 \\
15 \\
16 \\
17 \\
17 \\
18 \\
16 \\
17 \\
16 \\
16 \\
15 \\
16 \\
16 \\
16 \\
19\end{array}$ & $\begin{array}{l}6.8 \\
7.0 \\
7.0 \\
6.8 \\
6.6 \\
6.8 \\
6.0 \\
6.6 \\
5.6 \\
6.8 \\
6.2 \\
6.8 \\
6.6 \\
7.0 \\
7.0 \\
6.8 \\
6.8 \\
6.4 \\
6.6 \\
7.0 \\
7.0 \\
6.8 \\
6.6 \\
6.4\end{array}$ & $\begin{array}{l}1.013 \\
1.015 \\
1.020 \\
1.015 \\
1.010\end{array}$ \\
\hline
\end{tabular}

Eine Bestimmung des $\mathrm{rH}$ des Harns von 5 Schwangeren wurde $z u$ gleicher Zeit vorgenommen. Man ersieht aus Tabelle 2, daß hier auch das $\mathrm{rH}$ sich mit dem Fortschreiten der Tragzeit erniedrigt. Der Wert war besonders niedrig kurz vor der Entibindung.

Tabelle 3 zeigt ferner die Befunde des Harns von zwei Patientinnen mit 
Tabelle 2. rH des Harns von 5. Suhwangeren, zu gleicher Zeit bestimmt.

\begin{tabular}{l|c|c|c|c|c}
\hline Alter & $\begin{array}{c}\text { Schwanger- } \\
\text { schaftsmon. }\end{array}$ & $\mathrm{rH}$ & $\mathrm{pH}$ & sp. Gew. & Anmerkung \\
\hline 24 & 2 & 19 & 6.8 & & \\
37 & 2 & 20 & 6.8 & 1.010 & \\
26 & 8 & 17 & 7.2 & & \\
21 & 10 & 16 & 6.8 & & Kurz vor d. Gebrut. \\
23 & 10 & 15 & 7.2 & 1.006 & Kur
\end{tabular}

Tabelle 3. rH des Harns von Schwangeren mit Schwangerschaftsniere bzw. Eklampsie.

\begin{tabular}{|c|c|c|c|c|c|}
\hline Alter & $\begin{array}{l}\text { Schwanger- } \\
\text { rchaftsmon. }\end{array}$ & $\mathrm{rH}$ & $\mathrm{pH}$ & sp. Gew. & Anmerkungen \\
\hline $\begin{array}{l}35 \\
38\end{array}$ & $\begin{array}{l}8 \\
7\end{array}$ & $\begin{array}{l}17 \\
18\end{array}$ & $\begin{array}{l}6.8 \\
7.2\end{array}$ & $\begin{array}{l}1.008 \\
1.014\end{array}$ & Während per Kur. \\
\hline 26 & 9 & $\begin{array}{l}15 \\
18 \\
18\end{array}$ & $\begin{array}{l}5.6 \\
5.6 \\
6.0\end{array}$ & $\begin{array}{l}1.028 \\
1.022 \\
1.020\end{array}$ & $\begin{array}{l}\text { Kurz vor Kaiserschnitt. } \\
2 \text { Stunden n. d. } \\
\text { Operaion. } \\
2 \text { 'Tage n. d. Operation. }\end{array}$ \\
\hline
\end{tabular}

Schwangerschaftsniere im 8. und 7. Schwangersehaftsmonat mit deutlichem Ödem am ganzen Körper, Albuminurie und Blutdrucksteigerung und von einer an Eklampsie leidenden Patientin im 9 Sshwangersehaftsmonat mit Ödem, Albuminurie, Ascites, Oligurie und Krampfanfällen. Das rH des Harns ließ bei der Schwangerschaftsniere keinerlei Abweichung von dem der Giesunden erkennen. Demgegenüber war das rH bei der Eklampsie beträchtlich niedrig. Es war kurz vor der Entbindung durch den Kaiserschnitt 15, aber schon 2 Stunde nach der Operation 18.

\section{Auswertung der Ergebnisse.}

Das rH der Schwangeren senkt sich wenn auch nicht beträshtlich ganz allgemein in späteren Stadien der Gravidität. Das heißt, reduzierend wirkende Substanzen vermehren sich im Harn.

In der Tragzeit gehen bekanntlich Veränderungen des Stof fwechsels vor sich zugleich wird die Permeabilität der Niere gesteigert, so da $\beta$ also ungenügend oxydierte intermediäre Stof $f$ wechselprodukte in erheblicher Menge in den Harn eintreten können.

Im Harn der Schwangeren vermehrt abge: onderte, mehr reduzierend wirkende Substanzen sind Kreatin. Glukose, Allantoin. Urobilinogen und Indikan, ferner Harnsäure, Harnstoff, verschiedene Lipoide usw. Es ist bekannt daß 
sich Kreatin gegen Ende und Glukose schon im Frühstadium der Schwangerschaft im Harn vermehren. Die Mengenverhältnisse der anderen Substanzen im Harn der Sshwangeren sind je nach den Autoren vielfältig angegeben. Hier sei nur auf die Namen einiger Autoren (mit den Titeln der Aufsätze im Literaturverzeichnis in S. 353) hingewiesen: HYNEMAN (1912) und MATSU. MOTO u. TAHARA (1942) über Urobilin, RÜBSAMEN (1922) über Indikan im Blut (nicht aber im Harn) und DOI (1920) und FUJII (1941) über den Indikangehalt des Harns. Aus alledem scheint die vermehrte Absonderung von Kreatin und Glukose an dem Absinken des $\mathrm{rH}$ des Schwangerenharns den wichtigsten Anteil zu haben.

Bei der Sohwangerschaf isniere und Eklampsie war das Absinken des $\mathrm{rH}$ sehr augenscheinlich. BAR und DAUNAY (1908) bemerkten schon eine vermehrte Harnsäureausscheidung bei der Eklampiie. HYNEMAN (1912) konstatierte bei den Schwangerschaftstoxikosen die Vermehrung von Kreatin im Harn, und RÜBSAMEN (1922) wies im Harn der Schwangeren mit Nierenkrankheiten mehr Indikan als in demjenigen der gesunden Schwangeren nach.

Nach meiner Bestimmung des Harns war fast kein Untersehied des $\mathrm{rH}$ zwischen den Kranken mit Schwangerschaftsniere und den gesunden Schwangeren gefunden, aber bei Eklampsie erniedrigte sich das $\mathrm{rH}$ des Harns beträchtlich. In guter Übereinstimmung mit meinem Befund hat MIZUGUCHI (1943) auf eine abnorme Vermehrung des Jodatwertes des Blutserums bei der Schwangerschaftsniere und Eklampsie aufmerksam gemacht.

Zu bemerken ist schließlich daß die Wasserstoffionenkonzentration keine regelmäßigen Veränderungen während der Schwangerschaft anfwies. Das spezifische Gewicht des Harns sthwankte, senkt sich aber durchschnittlich etwas.

\section{Zusammenfassung.}

Um zur Histochemie indirekt beizutragen, wurde das Redoxpotential des Harns von 147 Schwangeren mit den Indikatoren bestimmt.

1. Das Redoxpotential des Harns fällt mit dem Fortschreiten des Schwangerschaftsmonats (d.h. die reduzierende Potenz der Harnsubstanzen als Ganzes nimmt zu), um schließlich unmittelbar vor der Entbindung am niedrigsten zu werden. Zahlenmäßig durch $\mathrm{rH}$ ausgedrückt: von 20 bis zu $16-17$.

2. Das Redoxpotential des Harns sank bei der Eklampsie bis zu rH 15 .

3. Die Wasserstoffionenkonzentration des Harns schwankte auch während der Schwangerschaft sehr, blieb aber durchsehnittlich unverändert. Das spezifische Gewicht sank durchschnittlich etwas. Diese Veränderungen stehen indessen mit dem Redoxpotentiale in keiner Zusammenhang. 


\section{内容自抄。}

妊婦 147 例に就いて尿の酸還電位を指示薬を用いて検査した。それとよ ると僅かの例外を除き，妊娠初期では $\mathrm{rH}$ 值は19-20で平常值に近く，妊 脄中期になると17一-18を示すものが多くなり，後期では压倒的に17-18の ものが增して来て，終期には16-17の低い值が多くなった，又妊娠肾の尿 の $\mathrm{rH}$ は健康妊婦のものとの間飞差は認められなかったが，子㾞では $\mathrm{rH}$

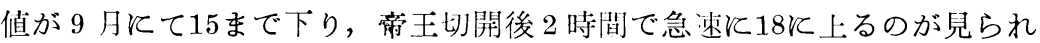

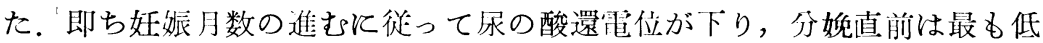
く，子㾞では更に強く下った。

尿の水素イオン湜度は妊拫時飞規則正しい変化を示さず，比重は屡々や や低下するが，これ等の変化は酸還電位のように娃娠経過と密接な関係を 持たなかった。

\section{Literatur.}

Bar u. Daunay : Über posteklamptische Harnsüurekrisen und deren Bedeutung. Soc. d'Obst. Paris. 1907. Zit. n. Zbl. Gynäkol. 32.-Doi, M. : Über die abnormen Komponenten des Harns im Gebiet der Geburtshilfl-gynäkologie, besonders über die Frequenz des Indicanuries. (Jap.) Acta gynaec. jap. (Nippon Fujinka Gk. Z.) 15 (1920). - H.jii, H.: Die Indicanreaktion im Gebiet der Geburtshilfl.-gynäkologie. (Jap.) Acta Obst. et Gynaec. 24 (1941). - Hyneman, Th. : Zur Frage der Leberinsuffizienz und des Kreatininstoff wechsels während der Schwangerschaft und bei den Schwangerschaftstoxikosen. Z. Gynäkol. 71 (1912). - Kusuhara, Y. : Eine Messmethode des Redoxpotentials von Mineralwässern und Körperflüssigkeiten mittels Indikatoren. Arch. hist. jap. 1 (1950). - Matsumoto, Y. u. T. Tahara: Über die indirekte Bilirubinreaktion des Serums und die Urobilinogenreaktion des Harns der Schwangeren. (Jap.) Acta Obst. et Gynaec. 25 (1942). - Mizuguchi, H : Studien über die Schwangerschaftstoxikosen. I. Schwangerschaftsniere und Nephritis. (Jap.) Acta Obst. et Gynaec. 26 (1943).Rübsmen, W.: Über die Bedeutung der Indicanämie und Hyperindican̈̈mie in der Schwangerschaft bei Nierenkranken und Nierengesunden. Arch. Gynäkol. 117 (1922). - Shimizu, Y.: Die Kolloidreaktion des Harns bei Schwangeren. (Jap.) Acta gynaec. jap. (Nippon Fujinka Gk. Z.) 44 (1949). 